\title{
La sombra de Bombay se proyecta en Davos 2004
}

Francisco Javier IbISATE

El eje del mal: la pobreza, el sida y la guerra

Después de escuchar en Davos los discursos de cuatro célebres críticos de la globalización -entre ellos, Joseph Stiglitz- los corresponsales de Le Monde concluyen que el actual eje del mal son la pobreza, el sida y la guerra. Algo está cambiando cuando dos de los codirectores del foro económico de Davos dicen que las tres palabras claves son 'cooperación, seguridad y prosperidad'. No es posible la seguridad sin prosperidad, ni la prosperidad sin seguridad y tampoco es posible la prosperidad ni la seguridad mundial sin la cooperación de todos. Los tres componentes del eje del mal generan la mayor inseguridad del mundo actual y una prosperidad truncada.

Hace dos años, el trigésimo segundo foro económico de Davos tuvo lugar en Nueva York ante los restos calcinados de las torres gemelas. Allí se habló de 'economía frágil, de inseguridad, de incertidumbre'; "la globalización de la ira se ha ido acelerando con el rápido crecimiento de las desigualdades". En ese escenario se relanza la guerra contra el terrorismo, la "seguridad duradera". Dos años más tarde, en Bombay se critica abiertamente y en Davos se acepta, en voz baja, la "inseguridad duradera". En el foro económico de Davos 2003 se propone un objetivo imposible: "construir la confianza" (building trust). Cómo construir la confianza si, primero en Davos, y luego en la sede de las Naciones Unidas en Nueva York la "coalición" había 
determinado, por propia autoridad, lanzar la guerra preventiva contra Irak, aduciendo razones que entonces y ahora se ha probado ser títulos colorados. El año 2003 ha sido el año de la desconfianza entre las potencias atlánticas y del mal llamado "choque de civilizaciones". Y para forzar la tuerza, el fracaso de Cancún.

En enero de 2004 se han roto dos tradiciones. Los foros mundiales de Davos y Porto Alegre no se han celebrado en la misma fecha, como se hiciera en los tres años anteriores. El cuarto foro social mundial no se ha celebrado en Porto Alegre, sino en Bombay. Se amplía la visión del mundo con un desplazamiento de los radares desde el área de las Américas hacia la zona más poblada del mundo: la India de los grandes contrastes. En el continente americano se habían sucedido una serie de cumbres, con saldo negativo: la cumbre de la OMC en Cancún, México; la quincuagésima octava asamblea general de Naciones Unidas, en Nueva York; la cumbre del ALCA 'a dos velocidades', en Miami, y la reunión de Monterrey, México, un acuerdo con desacuerdos para el alivio de la pobreza. El foro social de Bombay ha sido para muchas personas el descubrimiento de un nuevo mundo, comenzando por los 2.000 periodistas que, dicen, haber asistido. Esto ha permitido que del 16 al 21 de enero la atención se centrara en los problemas de la India, y en los problemas del mundo total desde una lectura india, para trasladarnos a continuación a la 'montaña mágica' de Davos y tener una mejor comprensión de los problemas en ambos mundos. La sombra de Bombay se ha proyectado sobre la blanca nieve de Davos.

\section{Bombay y Porto Alegre: dos foros distantes y distintos}

Desde 1970, el foro económico mundial tiene lugar en el balneario de Davos y siempre se reúnen los mismos 'amos del mundo'. El foro social mundial, nacido en Porto Alegre, este año se congrega en Bombay y se programa que en el 2006 se puede convocar en un país de Africa. Los objetivos son los mismos: presentar la globalización desde los sectores más inmersos en la sociedad civil y su participación activa en la formulación de un nuevo orden mundial. Cansados de andar protestando de cumbre en cumbre mundial, los inconformes con la 'globalización desde arriba' desean oponerse, no con un simple $\mathrm{NO}$, sino con el planteamiento de propuestas alternativas. Aunque el ideal y la esperanza son los mismos, aparecen sensibles diferencias 
entre los asiduos participantes en los tres foros de Porto Alegre y los congregados en Bombay.

De acuerdo a uno de los organizadores del foro en Porto Alegre, Cándido Grybowski, el $70 \%$ de los participantes habían frecuentado una universidad. La mayoría de los acreditados en Porto Alegre eran brasileños, un $51 \%$ eran mujeres y un $62,7 \%$ eran jóvenes (entre 14 y 35 años). "No esperábamos contar con un nivel tan elevado de escolaridad y una participación tan grande de jóvenes. Funcionamos como una escuela de formación ciudadana, lo cual supone una gran responsabilidad. Atraemos en su gran mayoría a jóvenes que no están adscritos a ningún partido político, pero nos preguntamos si hemos logrado darles respuestas". Grybowski siente que es difícil realizar una reunión mundial que represente a la sociedad civil del amplio Sur. Por esto el presente foro tendrá lugar en Bombay y la idea es que en el año 2006 se tenga en Africa. La Carta de principios del Foro Social Mundial excluye la participación de partidos políticos, en cuanto tales, así como de movimientos de lucha armada (ETA, FARC, entre otros) En Bombay se hará presente un foro paralelo (Bombay Resistencia) organizado por movimientos extremistas, que defienden la lucha armada. "Ellos dicen que nosotros somos neoliberales" (Le Monde, 08.01.04)

El escenario de Bombay es muy diferente. Para comenzar, el Partido de los Trabajadores, en el poder, subvencionó en gran parte el foro social de Porto Alegre, que tuvo lugar en la Universidad Católica de la PUC, mientras que en Bombay no se contó con la ayuda oficial del gobierno; la mayor parte del financiamiento estuvo a cargo de ONG extranjeras, como la Fundación Heinrich Böll, Oxfam International y algunas de las organizaciones indias participantes. No fue fácil distribuir a los más de 100.000 participantes en un antiguo local industrial, en un ambiente de ruido y polvo, donde vive buena parte de los 14 millones de habitantes de la capital. No fue tarea sencilla organizar los 2000 talleres y conferencias, así como instalar el sistema de traducción simultánea, dada la diversidad de lenguas presentes. El problema mayor era cómo integrar la atención a la diversidad de problemas de la India y naciones cercanas con el análisis general de la globalización.

Junto a la numerosa representación de las 135 organizaciones de la India, se han congregado 1.800 delegados venidos de Pakistán, unos 
mil de Bangladesh, 900 de Nepal, 600 de Sri Lanka, 430 japoneses y 370 sudcoreanos. Fuera de Asia, las delegaciones de Brasil (unos 6.000), de Alemania (2.000), de Francia (1.300), de Suiza (1.200), de Estados Unidos (unos 1.000), de Senegal (520), de Italia (350), de Bélgica (240), de España (230), más los 2.000 periodistas anunciados. Una variopinta Arca de Noé, cuya tripulación va a escuchar la voz de los más pobres de la inmensa India. Más del $80 \%$ son indios venidos del campo y de sectores urbanos marginados, a quienes se les ofrece la oportunidad de hablar abiertamente.

Los organizadores sienten que "es necesario un equilibrio entre la atención a los problemas locales y nuestra preocupación central que es la mundialización. A fuerza de celebrar la diversidad, que es un triunfo del movimiento, la misma diversidad puede hacerse menos comprensible. Pero no podemos ser elitistas. La cantidad tiene un efecto contagioso que permite dinamizar el movimiento e incorporar nuevas categorías de poblaciones. Es necesario encontrar un equilibrio entre el número y la acción. Lo cierto es que ellos representan en el mundo actual la única fuerza capaz de oponerse al sistema dominante". (Le Monde, 19.01.04)

Uno de los aspectos desconocidos para las delegaciones occidentales es que la sociedad civil de la India reagrupa a 135 organizaciones de sindicatos y partidos; la asociación de mujeres, All India Democratic Women, que lucha contra el fundamentalismo hindú, cuenta con siete millones de miembros. Las 2.000 conferencias y talleres reservan un amplio espacio a la intolerancia religiosa, a la exclusión que produce el sistema de castas y a la situación de la mujer. En la India son especialmente las mujeres quienes lideran estos grupos y movimientos sociales. Ahí se escucharán las voces de la escritora india Arundhati Roy, de la Premio Nobel de la Paz 2003, la iraní Shirine Ebadi y orras célebres figuras femeninas, que lanzarán el puente entre los problemas nacionales y la mundialización global.

\section{Los intocables, las mujeres y los excluidos de la sociedad india}

Un primer escándalo a denunciar es el sistema de castas, siempre presente, y la creciente discriminación de la mujer y de las minorías religiosas. Los intocables (dalits), casta inferior de la sociedad india, son la mayor parte de los 60.000 participantes del país anfitrión que quieren hacer oír su voz. Aunque oficialmente suprimido, el sistema 
de castas mantiene pleno vigor, entre ellos los 250 millones de dalits situados en la escala social inferior. "Nosotros tenemos dos mensajes: la dignidad humana es inviolable y la globalización neoliberal agrava la condición de los dalits". El proceso de liberación económica, emprendido por el gobierno en 1991, pesa sobre los dalits y las tribus. Por mandato constitucional se les habían adjudicado puestos de trabajo, de escasa calificación, en las instituciones estatales. Pero "el empleo de los dalits en el sector público ha disminuido mucho desde 1992". De 2,5 millones a 1,6 millones. La multiplicación de privatizaciones les deja también sin trabajo por los prejuicios aún vigentes en el sector privado. La modernización y mecanización de la agricultura acrece el problema. "Todas las tierras costeras han pasado a poder de multinacionales que crían camarones. Nos expulsan de nuestras tierras y de nuestro empleo". Los dalits son aptos para el trabajo agrícola, pero un $80 \%$ no tienen tierra. "Que se les devuelva la tierra". (Le Monde, 16.01.04)

Antonio Caño, enviado especial de El País, toca este problema en su artículo. "La antiglobalización se enfrenta a la pobreza". El foro social de la India se abre el mismo día en que el gobierno indio anunciaba la plena apertura del sector bancario al capital extranjero. Las nuevas políticas liberales han permitido que la economía crezca a un ritmo superior al $8 \%$ y ha engrosado una clase media que apoya estas reformas; ha supuesto también la reducción del inmenso aparato del Estado y el desempleo de quienes viven bajo su protección, particularmente de los dalits. "Casi la mitad de los tres millones de dalits han perdido sus trabajos en los nuevos tiempos de la liberalización, a los que se enfrentan en peores condiciones para competir, tanto por su inferior formación como por la discriminación de que son objeto". Casi la cuarta parte de este país está en las mismas condiciones de extrema pobreza.

"El problema de la extrema pobreza desborda cualquier otra de las reivindicaciones de la reunión -una larga lista que va desde la libertad para el Tibet, el combate contra el sida- y coloca inexorablemente al Foro frente a su mayor desafío: ofrecer una alternativa a la liberalización, o buscar una vía para humanizar el desarrollo. ¿Cómo se puede combatir la pobreza en países como la India sin recurrir a las vigentes reglas del mercado?. ¿Cómo es posible desarrollar sociedades postradas históricamente en la miseria sin exponerlas a la llegada del capital 
financiero, tan odiado en esta cumbre que hasta se prohibe el consumo de refrescos producidos por multinacionales? ¿Cómo hacer realidad el eslogan que afirma que "un mundo mejor es posible"? Los críticos de este movimiento se quejan de que el Foro ha tenido hasta ahora más éxito en las denuncias genéricas de los males del "liberalismo" y del "imperialismo" que en las respuestas a esas preguntas".

No significa esto que los cuatro foros sociales sean un tiempo y un derrotero perdidos, porque su primer estadio es el descubrimiento de la realidad mundial. "Los 70.000 participantes en la cumbre de Bombay intentan, no obstante, aportar algunas soluciones. Una de las dificultades para encontrar esas respuestas es la propia dispersión del movimiento antiglobalización. Bajo esta etiqueta se reúnen aquí cientos de organizaciones que oscilan desde el Falun Gong, un grupo espiritualista chino, hasta sindicatos europeos de la más pura tradición marxista. En medio quedan los grupos pacifistas, ecologistas y feministas, entre otros muchos". Antonio Caño dice que éste es el dilema de los organizadores: cómo dar respuesta a problemas tan distintos. Los mismos organizadores están divididos entre quienes quieren institucionalizar este movimiento y ofrecer una plataforma común y quienes creen que es mejor mantener el Foro como un espacio de reivindicación en el que se escuche una gran diversidad de opciones sin pretender acuerdos finales. Esta última es la línea dominante" (El País, 18.01.04).

Conviene recordar que en este foro se escucharán las voces de figuras internacionales como Arundhati Roy, Shirin Ebadi, Wandana Shiva, Mary Robinson, Joseph Stiglitz, Almed Ben Bella, Chico Whitaker y José Bové, que harán propuestas concretas y proyectarán en Davos la sombra de Bombay. La presencia de reconocidas figuras femeninas en este foro permite intercalar, en este mosaico de problemas, otra injusticia sociocultural, cuestionada ya en la cumbre de Beijing, celebrada en 1996, y más lacerante en todo el continente asiático.

\section{Los derechos de la mujer y la Corte Penal Internacional}

En Bombay se descubre la situación de las mujeres indias, "ciudadanas de segunda categoría", que quieren hacer oír su voz. A partir de unos breves testimonios, derivados del fundamentalismo hindú o de la ley islámica, se llega a una denuncia y a una propuesta concreta 
para la Corte Penal Internacional (CPI), encargada de juzgar genocidios y delitos de lesa humanidad.

"El consumismo alocado que se ha extendido en la sociedad hace que la dote que debe entregar la mujer sea cada vez más importante. Por ello, las familias prefieren el nacimiento de un niño que el de una niña. Se producen miles de abortos femeninos en la India. Miles de mujeres jóvenes son asesinadas por sus maridos o por sus suegros porque no pueden dar en dote el refrigerador o la televisión último modelo. La reducción de las subvenciones alimentarias y de ayudas a la educación perjudica a las mujeres. Si la familia no tiene suficiente dinero para mandar sus hijos a la escuela, sólo el niño irá. Lo mismo sucede con la alimentación: el niño comerá dos tiempos y la niña sólo uno". El foro social quiere "llamar la atención del gobierno y su responsabilidad ante estas atrocidades" (Le Monde, 16.01.04).

"El Foro de Bombay pide que la Corte Penal Internacional (CPI) se ocupe de delitos contra la mujer", escribe Antonio Caño. La Premio Nobel de la Paz 2003, Shirin Ebadi, respaldó el papel de la CPI como uno de los mayores símbolos de los esfuerzos por globalizar la justicia. "La Corte Penal Internacional es una prueba de que es posible una globalización con rostro más humano". Las reconocidas figuras femeninas, presentes en el foro, piden que la CPI tenga el respaldo de todos los Estados. "La Corte Penal Internacional es el primer mecanismo de derechos humanos que protege de forma práctica a los individuos por encima de los límites de los Estados". Amal Basha, presidenta de la organización de derechos humanos de Yemen, explicó que en el mundo árabe la lucha por los derechos humanos es algo muy minoritario y casi exclusivo de las mujeres. "El mundo árabe, dijo, está caracterizado por la cultura opresiva que margina sistemáticamente a gran parte de la población y especialmente a las mujeres, con el pretexto de la sharia (ley islámica)".

"Basha recordó que, de los veintidós países árabes que hay en el mundo, sólo dos, Jordania y Yibuti, han ratificado la Corte Penal Internacional. Tampoco Estados Unidos, Rusia o China (tres de los cinco miembros del Consejo Superior de la ONU) han ratificado todavía ese organismo". Todas estas figuras femeninas hacen un reclamo expreso: "que la CPI se ocupe cuanto antes de juzgar los delitos de violación, esclavitud, trata de blancas y otros que se cometen en forma masiva, según denunciaron las participantes, durante los con- 
flictos armados o por regímenes opresivos en distintas partes del mundo". La premio Nobel iraní recordó a las mujeres iraquíes "que sufrieron abusos y discriminación masivos durante el régimen de Saddam Hussein", y que, según ella, "no han visto todavía mejorar su situación durante la ocupación norteamericana". "Los derechos humanos no pueden imponerse por las armas", afirmó (El País, 21.01.04).

Tanto los organizadores como algunos de los reporteros presentes en Bombay temían, razonablemente, que este foro se centrara en problemas concretos del subcontinente indio. Sin embargo, a medida que avanzan las cinco jornadas aparece una doble novedad. Los problemas socioculturales de la India, los intocables, los excluidos y los derechos de la mujer, son lacras presentes, bajo otras modalidades, en muchas regiones del mundo. Los indígenas, la feminización de la pobreza, el mundo incierto del emigrante, la crisis moral del desempleo, las pandemias y, por desgracia, las matanzas étnicas, amenazan la seguridad de millones de excluidos. También en Bombay se han congregado, en cumbres paralelas, tres agrupaciones más radicales, que proponen la lucha armada contra "el imperialismo norteamericano". (Le Monde, 16.01.04) Se plantea así la doble tesis de la antiglobalización, vía lucha armada, y de la altermundialización, que pasa de la protesta a la propuesta de que otro mundo es posible. El Foro Social quiere apartarse de las llamadas "flores venenosas" que entorpecen las propuestas alternativas. Por esta razón el foro social de Bombay transciende de los desafíos locales al tema central de la globalización.

\section{Breve paréntesis: la economía de la India}

La crítica a la globalización necesariamente debía estar presente en el foro de Bombay, puesto que la India es un ejemplo típico del anverso y reverso de la economía de libre mercado, advirtiendo que muchos de sus éxitos y de sus debilidades son anteriores al modelo liberal. Como lo recuerda Cristophe Jaffrolet, desde 1950 la India opta por un modelo económico de sustitución de importaciones, sostenido por una planificación central que impulsa el desarrollo industrial interno al abrigo de la competencia internacional. Este modelo bastante cerrado y burocrático entra en un proceso de débil productividad, con baja competitividad de las empresas y con escasa participación en el comercio internacional. En 1980 se inicia una dolorosa apertura y gran prevención frente a las multinacionales, percibidas 
como un símbolo del imperialismo occidental. Esta modernización es financiada con un elevado préstamo, cuyo resultado será una gran deuda externa de $\$ 72.000$ millones en 1991 . La India es obligada a aceptar un plan de ajuste estructural del FMI, una de cuyas condiciones es la liberalización de la economía y su apertura a las empresas extranjeras.

La India se ha resistido a aceptar todas las recetas del FMI y su tendencia al neoliberalismo tiene un carácter relativo. El Gobierno mantiene un control de los porcentajes de inversión extranjera permitidos en las diversas ramas del sector real y de servicios, de suerte que la inversión directa extranjera (IDE) es diez veces menor que en China. Por ello, la India no sintió mucho la sacudida de la crisis financiera de 1997-1998. Bastantes multinacionales ven a la India como "un país taller", desde donde pueden exportar productos competitivos por el bajo costo de la mano de obra. La informática es uno de los sectores que ha progresado más, siendo la India el primer oferente de servicios en esta rama tecnológica. Su participación en el comercio internacional es todavía muy pequeña, $1 \%$, y el comercio exterior representa sólo el 9,8\% del PIB. Se puede decir que la India mantiene un control de la liberalización económica, y ello explica su fuerte oposición a los proyectos occidentales en la Organización Mundial del Comercio (OMC) y su alianza con China, Sudáfrica y Brasil en la cumbre de Cancún. Igualmente ha habido una fuerte resistencia a las privatizaciones de los servicios públicos, llamadas "desinversiones", y se generaron fuertes tensiones cuando, en 2003, se privatizaron dos de las mayores empresas petroleras.

Aunque la clase política esté convertida al liberalismo, ésta trata de ser prudente, porque muchos sectores dependen del Estado y no quieren enajenarse al electorado poniendo en la calle a un mayor número de desempleados, fruto de las privatizaciones. Las leyes sociales, que ofrecen garantía a los asalariados, no gozan de mayor amplitud y las garantías jurídicas ignoran al sector informal, restringidas a la aristocracia obrera de las empresas y a los empleados de cuello blanco. Se sigue protegiendo a bastantes talleres artesanales, que representan el $45 \%$ de la producción industrial. Esta política es criticada por economistas liberales que ven en ella un freno a la concentración industrial. Las estadísticas oficiales dicen que la pobreza se ha reducido "en cifras relativas" y que se ha elevado el PIB per capita en los diez últi- 
mos años. "Sin embargo, el país cuenta con el mayor número de pobres en el mundo (430 millones de personas viven con menos de un dólar, según en Banco Mundial). Además, sigue descendiendo su índice de desarrollo humano, de acuerdo al informe del PNUD); ha pasado del puesto 94 (sobre 130 países) en 1994, al 124 en 1995 y al 127 en 2003. Esta clasificación refleja el crecimiento de las desigualdades" (Le Monde Diplomatique, 16.01.04). Esta India de los grandes contrastes ha sido el escenario propicio para que representantes de organizaciones nacionales y reconocidas figuras internacionales plantearan serias críticas y también propuestas concretas de cara a la globalización imperante.

\section{Cuestionamiento de la globalización económica}

Antonio Caño, en su artículo: "La antiglobalización se enfrenta a la pobreza", dice que el foro de Bombay comenzó denunciando algunas injusticias presentes en las relaciones Norte-Sur. "Llamémosle como le llamemos, imperialismo, neoliberalismo o fundamentalismo de libre mercado, estamos ante la misma realidad: un mundo injusto y unas relaciones injustas", afirma Juan Somavia, de la Organización Internacional del Trabajo (OIT). Según Somavia, "desde que comenzó el proceso globalizador, a principios de los noventa, sólo en América Latina hay un $50 \%$ más de parados, un $50 \%$ más de trabajadores en la economía informal y el poder de la media del salario mínimo se ha reducido un $25 \%$. Esto es éticamente inaceptable y políticamente insostenible". Un hecho decisivo de esta injusticia, dicen varios participantes, fue el nacimiento de la Organización Internacional del Comercio (OMC) en 1995, creada para propiciar el desarrollo a través del intercambio comercial; pero ha servido a todo lo contrario. "Nueve años después del nacimiento de la OMC hay cien millones más de pobres en el mundo", asegura el activista filipino Walden Bello. El sindicalista francés José Bové y la ecologista india, Wandana Shiva sostienen que combatir la OMC es la prioridad para los enemigos de la globalización. El fracaso de Cancún es la prueba de la injusticia de su causa. "La crisis de la OMC, a raíz del fracaso de Cancún, es una de las principales muestras del fracaso de la globalización".

El foro no está en contra del comercio, pero quiere propiciar un comercio justo, "en el que los productores de los países pobres tengan acceso a los mercados de los países ricos en las mismas condicio- 
nes en que los productos de los Estados desarrollados penetran en los mercados del Sur". Oxfam calcula que "los agricultores de los países pobres pagan un $400 \%$ más de aranceles cuando exportan a los países ricos que los agricultores de los países ricos pagan para vender a los pobres". Una vez más, igual que en las OMC de Seattle, Doha y Cancún, el Foro pide la eliminación de las subvenciones agrarias en Estados Unidos y Europa, "que hacen ruinosa la producción de algunos de esos bienes en el tercer mundo. Se agregan otros daños, como la destrucción de las estructuras de control en los países subdesarrollados. Por ello, hay que luchar en tres frentes: la reconstrucción de los poderes locales, que son los verdaderos generadores de riqueza; el fortalecimiento de los Estados, sobre bases democráticas, para hacer frente a la globalización, y por último, la reforma de las organizaciones internacionales para democratizar la toma de decisiones". Este es un momento de decisión para el Foro. (El País, 18.01.04)

En bastantes intervenciones los organizadores y los delegados presentes en el foro han planteado un interrogante sobre los resultados prácticos que puedan lograrse en Bombay. A modo de ejemplo, Mari Takenuchi, representante de la Confederación Japonesa de Ferrocarriles, dice: "Hemos realizado muchos contactos que nos permiten crear una red internacional. Pero todavía no tenemos soluciones concretas. Nos hace falta un plan de acción". Le Monde, 21.01.04) Esta formulación del problema se repite muchas veces y ha sido recogida por el Premio Nobel de Economía, J. Stiglitz en la entrevista concedida a Laurence Caramel, enviado especial de Le Monde. Trasladamos unos párrafos de esta entrevista.

"Usted ha intervenido el lunes 19 de enero en el Foro Social de Bombay. ¿Acaso eso significa que Ud. respalda el movimiento altermundista"?, interroga Caramel. Responde el entrevistado: "El foro social es un acontecimiento muy positivo. Es mucho lo que ha sufrido el mundo con esa idea, según la cual no habría mas que una manera de alcanzar el crecimiento económico, de desarrollarse, de que no hay sino una sola manera de organizar la sociedad y la economía. Las discusiones aquí tratan, por el contrario, de demostrar que existen varias vías y también múltiples objetivos. Ellas dejan al descubierto a qué punto la focalización sobre el crecimiento es excesiva. Creo que puedo ayudar en este contexto, puesto que es necesario reformar la globalización. El proceso del Foro puede contribuir a ello de manera 
efectiva. Los 100.000 participantes van a regresar a sus países y, a través de sus redes, hacer que progresen un cierto número de ideas. Una sociedad civil mundial se está construyendo, ella tiene un impacto. Por su cuestionamiento de las instituciones, su trabajo de análisis, ella ha acelerado, por ejemplo, la toma de conciencia del vínculo que existe entre la globalización y el crecimiento de las desigualdades".

Esta primera cita de Stiglitz recoge los sentimientos expresados en la ceremonia de clausura del foro social mundial. (Headlines 2004/ India WSF) $\mathrm{Ni}$ en el foro social de la India, ni en el foro económico en Suiza se saca un documento final con políticas económicas concretas, universalmente válidas, "aunque destacan los avances, en términos más de fuerza social que de propuestas innovadoras, de los asambleístas rebeldes frente a la mundialización respecto a los de los integrados en ella, aún con atisbos de mala conciencia". (El País, 24.01.04) En realidad, los dos foros, social y económico, resultan estar cada vez más relacionados. En enero 2003 Lula da Silva habló en Porto Alegre y en Davos y este año J. Stiglitz habló en ambos foros. Por eso podemos decir que la sombre de Bombay se ha proyectado sobre la blanca nieve de Davos.

En lo que se refiere al plan de acción no se parte de cero. En el foro social de Porto Alegre, 2002, se presenta un programa de análisis y de acción asentado en cuatro ejes mayores: "1) La producción de riqueza y la reproducción social. 2) El acceso a las riquezas y la sustentabilidad. 3) La afirmación de la sociedad civil y los espacios públicos. 4) Poder político y ética en la nueva sociedad". Cada uno de estos ejes analiza cómo juegan los motores de la globalización 'desde arriba' y cómo debe erigirse la globalización 'desde abajo'. (Entorno económico mundial. UCA Editores 2003, pp. 236-239. ECA 2003; pp. 560-561) Al leer los temas incluidos en estos ejes apreciamos el cúmulo de datos y de experiencias con que Bombay enriquece a Porto Alegre. Para plantear una alternativa es menester comenzar por analizar cómo funciona la globalización, conocer sus logros y resaltar sus desmanes económicos, sociales, culturales, humanos.

Desde esta perspectiva es aleccionadora la ya citada entrevista concebida que Stiglitz concedió a Caramel (de aquí en adelante, J.S. y L.C.): "Los altermundistas hacen un análisis totalmente negativo de la mundialización, y en particular para los países emergentes o en vías de desarrollo. ¿Comparte Ud. ese análisis?". (J.S.): "La realidad es 
mucho más compleja. Países como la India muestran como, por una parte, la mundialización puede ser un formidable vector para el crecimiento y, por la otra, dejar a una gran parte de la población abandonada en el olvido. Desde hace veinte años, mucha gente ha sufrido de ese proceso de liberalización. Mucha más gente aún simplemente no ha visto que su vida cambie, mientras que se les había prometido un mundo mejor. Y se preguntan por qué, mientras que la prioridad absoluta era el crecimiento económico con detrimento de consideraciones de orden social o políticas; ese objetivo (el crecimiento) ni siquiera se ha alcanzado. La otra crítica que se hace se centra en el "déficit" de democracia, de participación, que caracteriza a la mundialización en lo relativo a la toma de decisiones a escala internacional. Esto es muy importante por cuanto sólo un proceso de toma de decisiones abierto a diferentes perspectivas, diferentes preocupaciones, puede conducir hacia un modelo de democracia duradera. En lugar de ello, no tenemos en la cúspide de las instituciones internacionales mas que a tecnócratas, ministros de finanzas y de comercio y presidentes de bancos centrales".

(L.C.): " ¿La mundialización se sustenta sobre la voluntad imperialista de EEUU, como se ha denunciado sin tapujos aquí?".

(J.S.): "El concepto de imperialismo es muy amplio. Si se trata de decir con ello que hemos entrado, con referencia al siglo XIX, a una forma de colonialismo, debo decir que no. Sin embargo, es cierto que EEUU utiliza su supremacía económica y militar para orientar al mundo hacia el servicio de sus intereses, y ello en detrimento de otros países. Esto es tanto más verdadero desde la elección de George W. Bush. El presidente habla mucho de democracia. Pero el unilateralismo no es compatible con la democracia, y esto plantea un auténtico problema del que tenemos varios ejemplos a través del funcionamiento de instituciones como el Fondo Monetario Internacional o la Organización Mundial del Comercio".

(L.C.): "Hay, por lo menos, tres reivindicaciones sobre las cuales los altermundialistas están de acuerdo: la anulación de la deuda externa de los países pobres, la creación de una cuota ('tasa') global para financiar el desarrollo y la suspensión de las privatizaciones. ¿Qué opina de ello?". 
(J.S.): "Sobre el primer punto, es cada vez más evidente que los diferentes mecanismos de reducción o de condonación de la deuda no han sido suficientes. El caso de Irak pudiera hacer evolucionar la situación, estableciendo una especie de jurisprudencia. Si se determina que los iraquíes no deben pagar la deuda acumulada por Saddam Hussein, entonces muchos otros países podrán también hacer valer que no tienen por qué asumir políticas pasadas de sus déspotas, por lo que no deberán honrar esas deudas "horrorosas. En cuanto al segundo punto, me parece indispensable conseguir una fuente de financiamiento a escala mundial, no sólo para conseguir recursos para el desarrollo, sino también para preservar los bienes públicos mundiales, tales como el medio ambiente, la salud... Hay varias proposiciones sobre la mesa. La Tasa Tobin tiene al menos la virtud que debiera tener cualquier impuesto. Gravaría algo nocivo: el flujo de capitales especulativos que contribuyen a la volatilidad de la economía mundial. Pero para serle sincero, no sé cómo es que se podría instrumentar".

"En fin, sobre las privatizaciones, la experiencia nos muestra pocos éxitos y muchos fracasos. En América Latina hemos podido comprobar como las privatizaciones no han aportado nada cuando los servicios públicos funcionaban bien; pero, por el contrario, en empresas deficitarias la intervención del sector privado más bien sirvió para mejorar las cosas. El problema radica en que los gobiernos siempre quieren privatizar lo que funciona puesto que ello les garantiza la venta. El agua es notablemente un sector en el que las privatizaciones casi siempre han fracasado, porque es muy difícil que haya competencia en un sector de esa naturaleza. Lo que han hecho las privatizaciones es sustituir un monopolio público por otro privado, sin beneficio alguno para la población".

En esta entrevista, Stiglitz resume algunas reflexiones vertidas en el capítulo primero ("La promesa de las instituciones globales") de su obra "El malestar en la globalización". Dos frases muestran su apoyo a estos foros sociales: "Las protestas han provocado un enorme caudal de exámenes de conciencia desde el poder político". Incluso los políticos conservadores, como el presidente francés, Jacques Chirac, han manifestado su preocupación porque la globalización no está mejorando la vida de quienes más necesitan de sus prometidas ventajas".(p. 28) "Sin alternativas, sin vías para expresar su inquietud, para instar a un cambio, la gente se alborota. Es evidente que las calles no son el 
sitio para discutir cuestiones, para formular políticas o anudar compromisos. Pero las protestas han hecho que funcionarios y economistas en todo el mundo reflexionen sobre las alternativas a las políticas del Consenso de Washington en tanto que única y verdadera vía para el crecimiento y el desarrollo" (p. 46).

Un detalle interesante es el hecho que J. Stiglitz haga referencia a la deuda externa acumulada por Saddam Hussein, y que la jurisprudencia pueda determinar que los iraquíes no la deban pagar. En el foro de Porto Alegre 2002 se constituyó un tribunal de seis miembros, entre ellos el Premio Nobel de la Paz, Adolfo Pérez Esquivel, El veredicto se expresó en estos términos: "La deuda de estos países es ilegítima, injusta e insostenible", incluso "odiosa": una noción de derecho internacional que existe desde 1920: "un pueblo puede rechazar el pago de una deuda de un régimen anterior, si se prueba que el gobierno la utilizó para beneficio propio". (Ibidem; p.238) Como se está analizando jurídicamente la "deuda odiosa" del Irak de Saddam Hussein (\$130.000 millones), Stiglitz deduce: "entonces otros países podrán también hacer valer que no tienen por qué asumir políticas pasadas de sus déspotas, por lo que no deberán honrar esas deudas horrorosas". Este tema se trató en la cumbre de Monterrey ("El alivio de la pobreza"), marzo 2002. (Ibidem; pp. 239-248) También ésta es una propuesta importante.

Stiglitz hace una breve referencia a la "Tasa Tobin", que gravaría los flujos especulativos "que contribuyen a la volatilidad de la economía mundial". Estas tasas servirían a crear un fondo para el desarrollo mundial. Aunque difícil de instrumentar, la propuesta aparece -de una u otra manera- en varios autores luego de la crisis financiera mundial de 1997, entre ellos George Soros, Dani Rodrink, J. Stiglitz, Paul Krugman, Paul Samuelson. (Ibidem; pp. 139-142) Lo importante es que Lula da Silva en Davos 2003 y junto con Jacques Chirac en Davos 2004 están proponiendo crear un fondo especial "contra el hambre" a partir de "taxas" sobre algunas ventas de armas y ciertas transacciones financieras. (Le Monde, 31.01.04) El reconocido historiador Paul Kennedy, luego de recordarnos la parábola de Buen Samaritano, invita a los 'ávidos consumistas' del mundo desarrollado a que retiren "un diezmo" de sus gastos y entregarlo a una de las reconocidas ONG (Oxfam o Cafod, por ejemplo) para ayudar a los países más necesitados. Esta sería, al menos, una forma de "tranquilizar sus 
conciencias". ("El escándalo de la pobreza mundial". El País, 23.01.04) También ésta es una propuesta realista que va tomando cuerpo en algunas cumbres mundiales.

En la entrevista a J. Stiglitz se deja entrever que en Bombay, al analizar el comportamiento de la globalización, se criticó al imperialismo norteamericano y de manera concreta la invasión a Irak. Se discutió en algunos seminarios el tema del militarismo, la guerra y la paz y los oradores declararon "la guerra al imperialismo norteamericano". "La mayor amenaza al mundo viene de los Estados Unidos y de su presidente Bush. Si Bush permanece en el poder en 2005, el mundo asistirá a otra guerra en Corea", dijo el pacifista surcoreano Keun Soo Hong. La crítica más dura provino, al parecer, de la Premio Nobel de la Paz, Shirin Ebadi: "La invasión norteamericana es una de las cuestiones más importantes para este foro. Norteamérica, aduciendo la falta de democracia, ha atacado Irak, pero no se puede exportar la democracia. No se pueden exportar los derechos del hombre con bombas". Shirin Ebadi pidió que Estados Unidos y los países que apoyaron a Saddam respondan ante el Tribunal Penal Internacional. "Debemos enviar a Saddam Hussein al Tribunal Penal Internacional por los crímenes que cometió contra su propia nación, pero también contra la mía. Pero Estados Unidos y los Gobiernos europeos colaboraron con él y son responsables y deben ser sentenciados en el mismo banquillo". Estos breves testimonios muestran la aversión a una globalización que suma al imperio económico "el derecho de la fuerza militar".

El mismo día en que se clausura el foro social de Bombay, el 21 enero de 2004, se inicia el foro económico en Davos. Al ser ambas reuniones foros mundiales sus agendas presentarán una relativa cercanía, no sólo porque la guerra en Irak es un punto de interés común, sino porque también lo es la globalización en cuanto globalización, es decir, en cuanto la frustración y la inseguridad de los congregados en Bombay hace imposible la prosperidad y la seguridad de los reunidos en Davos. En otras palabras, que es necesario pasar de un mundo unilateral a una acción multilateral gracias a la cooperación de todos. El mundo está interrelacionado no sólo por el Internet, sino por problemas comunes a todos los bloques de naciones. En teoría, este era el mensaje principal que se ha querido transmitir en Davos. 


\section{La clave de Davos: "cooperación, seguridad y prosperidad"}

Philipe Bourguignon y Thierry Malleret, codirector general y director del foro de Davos respectivamente, afirman que el desafío mundial requiere integrar estos tres objetivos claves. Vivimos en un mundo donde "no es posible la seguridad sin la prosperidad, ni la prosperidad sin la seguridad; ambos objetivos son inseparables. Igualmente, los problemas globales no pueden resolverse mas que de una manera global, es decir, incluyendo a todos los actores claves en la búsqueda de soluciones. De ahí la idea simple de la 'inseparabilidad' de las tres nociones; en esta correlación triangular, la ausencia de uno de los tres ingredientes compromete la misma idea de progreso. ¿Por qué?”. Es claro que vivimos un sentimiento de inseguridad y que, a ejemplo del 11 de septiembre, pueden repetirse graves incidentes de amplia repercusión. La mayoría de países desarrollados están pagando una "sobretasa Bin Laden", que se traduce en miles de millones de euros, gastos militares, millones de horas perdidas en los aeropuertos a causa de los mayores controles de seguridad. Muchas industrias y servicios sienten el azote del terrorismo, al que se suman más de quince conflictos entre Estados, docenas de guerras civiles y larvadas luchas interétnicas. Lo novedoso es que estos directores del foro económico actualizan las "amenazas duras" junto con las "amenazas blandas" que Kofi Annan quiso que fueran agenda de preocupación en la $58^{2}$ asamblea de Naciones Unidas, septiembre 2003, y que ahora pasarían a ser puntos de agenda central para los congregados en Davos. Transcribimos algunos párrafos de este documento.

"La seguridad debe entenderse en un sentido amplio, lo que $\mathrm{Na-}$ ciones Unidas llama la "seguridad humana". Los desafíos provocados por el hambre, la pobreza, toda clase de tráficos, la ausencia de un sistema de educación, de salud, o la falta de libertad son realmente gigantescos. Citando sólo unos ejemplos: 800.000 personas murieron el pasado año en conflictos bélicos; 22 millones murieron por falta de cuidados sanitarios y 800 millones siguen sufriendo de hambre. Más de 42 millones de personas padecen el sida y serán 100 millones en 2005. A todos interesa mejorar la "seguridad humana" porque es ahí donde se presenta la relación mayor entre seguridad y prosperidad. $\mathrm{Si}$ hoy día 1.800 millones viven con menos de un dólar al día, ces sólo problema de ellos? No, es un problema de todos. Sin tomar en cuenta la obligación moral, es conveniente que los países ricos ayuden a los 
países pobres. ¿Por qué? Porque vivimos en una aldea global cada vez más interdependiente. La pobreza, la frustración de los demás se convierte en problema nuestro: reducción del mercado a nuestros productos y servicios, incremento de la emigración ilegal, mayor contaminación ambiental, enfermedades contagiosas, fanatismo y terrorismo".

"Es necesario dar un sentido más amplio al concepto de prosperidad: no sólo la capacidad que tienen los países desarrollados para mantener un ritmo normal de crecimiento, sino también en el sentido de compartir en forma equitativa los frutos del crecimiento, de un mecanismo capaz de abolir la aterradora fosa que separa los países pobres de los países ricos. Esto no se realiza de forma espontánea porque, en contra de la teoría en boga, los países ricos se enriquecen más rápido que los países pobres. En resumen, partimos desde la economía y caemos necesariamente en la seguridad, que se mide por nuestra capacidad de dar respuestas a una multitud de nuevos desafíos como el síndrome respiratorio, el terrorismo, las pandemias; uno de los efectos de la globalización es que se ponen al descubierto estos problemas que parecían estar controlados. El Internet, el celular, los aviones reducen el mundo y nos dan el sentimiento de cercanía".

“¿Cómo resolver estos problemas globales, cómo mejorar al mismo tiempo la seguridad y la prosperidad?. Con la cooperación. No hay otra solución, porque nadie, ningún grupo, ningún país, ninguna institución dispone de medios y de la necesaria legitimidad para llevar a término, por sí misma, esta tarea. Han pasado los tiempos en que los gobiernos creían poder resolver ellos solos los problemas del mundo, mientras que los negocios se concentraban en sus contabilidades y la sociedad civil se dedicaba a criticar sin aportar soluciones concretas. Hoy día, la búsqueda de soluciones viables exige una forma de gobierno en red; en otras palabras, gracias a la cooperación entre los negocios (instituciones que crean el valor), la política (gobiernos y parlamentos) y la sociedad civil ampliada: ONG, líderes sindicales, religiosos y académicos. Integrar los negocios y la sociedad civil es, ante todo, ampliar la representatividad y con ello la legitimidad, algo muy necesario porque el mundo de la empresa y la sociedad civil son verdaderos actores mundiales, a diferencia de los gobiernos que tienen el poder de legislar, pero actúan localmente..." Esta es la razón por la que se ha reunido el foro económico en esta Montaña Mágica 
del 21 al 25 de enero, con la presencia de 2.000 responsables de las grandes tomas de decisiones. Es una idea simple pero ojalá muy eficaz para elaborar en común las soluciones las soluciones a los problemas del mañana. (Le Monde, 21.01.04)

\section{Los negocios van bien; el resto, mal}

Con el repunte de la economía norteamericana en el tercer trimestre de 2003 vuelve a subir el termómetro de la confianza en el foro de Davos, pero se trata de una prudente confianza. "Luego de dos años de purga debida a la explosión de la burbuja, los beneficios vuelven a subir. Recuperación de la economía mundial. El fuerte auge de Estados Unidos arrastrará también a Asia, América del Sur, Japón y Europa. La expansión alcanzará ritmos del $4 \%$ al otro lado del Atlántico, $6 \%$ en Rusia, $6 \%$ en la India y $8 \%$ en China". En terminología de Davos: "las perspectivas de consolidación global continúan mejorándose". No se teme un crack del dólar porque el papa Alan Greespan ha dicho que no siente temor por el financiamiento futuro de los déficits norteamericanos. (Le Monde, 17.01.04)

Pero no todos piensan lo mismo. Jacob Frenkel, de Merryll Linch, economista presente en Davos 2003, "expresó su confianza en la recuperación de Estados Unidos, pero puso algunos reparos". Steffen Roach, de Morgan Stanley, también presente en Davos 2003, concretó algunos de los reparos: "Si se miran las estadísticas de la productividad, no se puede menos que espectacular. Pero ahora tenemos un nuevo paradigma: persiste el desempleo. En esta recuperación, la economía mundial depende de un solo motor y Estados Unidos padece unos déficits crecientes, niveles de endeudamiento récord de las familias y una inusual falta de crecimiento de empleo que afecta los ingresos de la gente. Hoy lo que tenemos es una sustitución de elevados salarios en el mundo desarrollado por productos y servicios producidos con bajos sueldos en países como la India".

Varios economistas, como Laura D'Andrea, de London School y ex asesora de Clinton, se preguntan si "el resto del mundo seguirá dispuesto a prestar a Estados Unidos en el futuro y en qué condiciones". Kennet Rogoff, anterior jefe de economistas del FMI, afirma que "el dólar tiene que caer un $15 \%$ más del $14 \%$ que ya ha descendido en términos comerciales, si se busca reparar el déficit por cuenta corriente de EEUU y reducirlo al 1,5\% del actual 5\% del PIB" ${ }^{n}$. Rogoff 
no comparte la promesa de Bush sobre la reacción del mercado laboral con crecimientos importantes en los próximos meses. "Eso es pura política. Los economistas nunca hemos sabido cuál es la tasa de desempleo medio compatible con la inflación y un crecimiento fuerte en Estados Unidos. Los años noventa fueron una excepción. Bush ha conseguido la mejor recuperación que se puede comprar. Y yo creo que es sólida aun cuando no se vayan a crear los millones de puestos de trabajo que promete". Los empresarios europeos no se quejan sólo de la caída del dólar, sino de que la han acompañado las monedas de las economías emergentes asiáticas, a comenzar por China. El peso del ajuste en los tipos de cambio recae casi exclusivamente sobre los europeos". (El País, 22.01.04)

Sin abundar en más testimonios sobre el anverso y reverso de la posible recuperación económica, se respira un sentimiento común de que no puede haber prosperidad sin seguridad. Un sondeo realizado por la Gallup, a petición de los organizadores del foro, muestra que la mitad de los encuestados cree que la seguridad del mundo es "débil" y que la siguiente generación vivirá en un mundo menos seguro. Lo que alarma a los 'líderes del mundo' es que la inseguridad ensombrece su vida económica y el porvenir de sus hijos. "Está claro, la prosperidad no camina sin la seguridad. Como la situación iraquí no parece mejorar, como las guerras se suceden en el Sur y como el Norte vive cada día bajo 'niveles de alerta' del terrorismo, el sentimiento general se degrada. Un hombre de negocios inteligente lo comprende fácilmente: su negocio se verá afectado en cualquier momento. Por ello, se realizó un nuevo sondeo: ¿las autoridades políticas hacen un esfuerzo por crear la seguridad en el planeta?. La respuesta es claramente 'negativa'. La seguridad es un todo que incluye un conjunto de condiciones: la paz, pero también la lucha contra el hambre, contra la pobreza (causa de los extremistas) y a favor de la educación, de la salud, del medio ambiente y de los derechos humanos. La economía se recupera, pero el resto anda mal". En la óptica de algunos asistentes a Davos se enciende un peligro: "Nosorros, los patronos, hacemos nuestro trabajo; ustedes los políticos hagan el suyo". Esta posición parece olvidar que de las relaciones económicas y laborales emanan las desigualdades y la inseguridad". (Le Monde, 17.01.04)

Si Kofi Annan habló de las amenazas 'duras y blandas' en la cumbre de Nueva York, también lo hizo en Davos. El clima económico y 
la seguridad son menos favorables al mantenimiento de un orden global estable, equitativo y basado en Reglas. El fracaso de las negociaciones comerciales en Cancún y otros retrocesos o falta de avances ponen la globalización en entredicho. Está sacando a cientos de millones de persones de la pobreza, especialmente en China y en la India, pero ha condenado a otros tantos a la miseria. No hay duda sobre las consecuencias positivas, sino sobre sus "severas consecuencias negativas". El Secretario General de Naciones Unidas solicitó a los países ricos "abrir enteramente sus mercados a todos los productos de los países en desarrollo y a eliminar todas las subvenciones que imponen a estos países una competencia desleal". También pidió al sector privado que ayude a paliar el problema del hambre en muchas regiones del mundo, y el magnate de la informática, Bill Gates, ofreció donar, en cinco años, mil millones de dólares al Programa de la ONU para el desarrollo. Annan agregó: "el terrorismo separa a los pueblos, pero la guerra contra el terrorismo puede exacerbar estas divisiones". Otros asistentes a Davos, luego de escuchar el discurso del vicepresidente $R$. Cheney, llegaron a la misma conclusión: "el resultado de la guerra contra el terrorismo es más guerra y más terror". (El País, 26.01.04)

\section{Irak en el centro de Davos}

Lo que comenzó mal continúa peor, alimentando la inseguridad mundial. En el foro de Davos 2003 se hicieron presentes Colin Powell, secretario de Estado, y Paul O'Neil, entonces secretario del Tesoro. En Davos 2004 asisten Richard Cheney, vicepresidente, y John Ashcroft, ministro de Justicia. En menos de un año los dos primeros hablan otro discurso. C. Powell había asegurado que Saddam Hussein mantenía cercanas relaciones con el grupo Al Qaeda y que Irak desarrollaba armas de destrucción masiva, entre cien y quinientas toneladas de agentes químicos. Ahora C. Powell ha admitido "que la presencia de armas de destrucción masiva es una cuestión abierta. Todavía no sabemos. Teníamos preguntas que debíamos aclarar", dando a entender, en lenguaje diplomático, que se fiaban de los informes que habían recibido y que, de acuerdo a expertos, Rumsfeld y Wolfowitz habían inflado. No había armas de destrucción masiva, ni la amenaza era inmediata, ni existían vínculos entre Bagdad y al Qaeda. "Y ahora sale a la luz que Bush se propuso forzar un 'cambio de régimen' en 
Irak siete meses antes del 11-S de 2001. Poco a poco las sospechas de las mentiras sobre las que se montó la guerra se van tornando en confirmaciones". (El País, 16 y 25.01.04)

Paul O'Neil, primer secretario del Tesoro de George W. Bush, ha afirmado que "la caída del presidente de Irak, Saddam Hussein, era prioridad de la presidencia, enero 2001, ocho meses antes de los atentados del 11 septiembre", en revelaciones hechas al periodista Ron Suskind, cuyo libro titulado El precio de la lealtad, aparecerá próximamente. Paul O'Neil tuvo acceso a un documento, calificado de 'secreto': "un plan para Irak después de Saddam", discutido desde enero 2001. La Casa Blanca anunciaba planes de despliegue de fuerzas de paz, tribunales de guerra y también de distribución de las riquezas petroleras iraquíes". O'Neil decía en el diario Time que "nunca vio en los datos de este informe una verdadera prueba" de la presencia de armas de destrucción masiva en Irak. "Había algunas afirmaciones y opiniones expresadas por algunas personas, pero yo no nací ayer, y conozco la diferencia entre una prueba y una opinión, ilusión o conclusión que puede sacarse de algunas presunciones". Estas confidencias del anterior secretario del Tesoro coinciden con los informes de la Fundación Carnegie para la paz internacional y de Jeffrey Record, del Colegio de guerra de la fuerza armada, según los cuales Bush había exagerado la amenaza iraquí, dando inicio a "una guerra preventiva inútil". (Le Monde, 8 y 13. 01.04)

A estos informes se agrega el testimonio de David Kay, responsable de la misión de 1.400 especialistas enviados a Irak en junio de 2003 para investigar la presencia de armas de destrucción masiva, puesto que los inspectores de Naciones Unidas no las habían hallado. "Creo que no existían tales armas", dijo al renunciar a su cargo. Esto ha sido un duro golpe para Bush, quien en reciente discurso a la nación había dicho: "El informe Kay ha identificado docenas de actividades relacionadas con programas de destrucción masiva y una significativa cantidad de equipo que Irak ocultaba a las Naciones Unidas. Si no hubiéramos actuado, esos programas de armas destructivas del dictador se estarían desarrollando hoy". No fue éste el informe de Kay: "Esto de que habla todo el mundo son los inventarios producidos luego de terminada la guerra del Golfo, 1991, y no creo que haya habido un programa de producción de cierta envergadura en los años 
1990. Creo que tenemos la prueba manifiesta de que (los iraquíes) no han relanzado una producción mayor, y esto es lo que testificamos".

Esta confluencia de testimonios sirve para que la oposición demócrata devuelva al bumerán al campo republicano. El senador John Rockefeller dijo: "Es cada vez más evidente que los informes sobre las armas iraquíes eran falsos" y que la administración republicana "ha exagerado la amenaza nuclear (de Irak) y los lazos (de Bagdad) con la red terrorista Al Qaeda. El resultado es que Estados Unidos debe pagar un elevado precio". También el primer ministro británico Tony Blair ha pagado un elevado precio a causa del suicidio de David Kelly, experto en armas de destrucción masiva, quien había afirmado que el gobierno británico había exagerado la amenaza iraquí". (Le Monde, 24.01.04) El testimonio de P. O’Neil, al afirmar que la invasión de

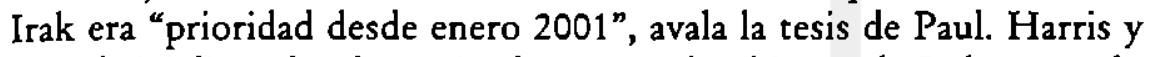
Joseph Stiglitz: el 6 de noviembre 2000 el gobierno de Irak, segundo productor mundial de petróleo, trasladó sus multimillonarios activos en dólares a la zona euro. ¿Qué pasaría si todos los miembros de la OPEP imitan a Irak? "Se arma la gorda", dijo Stiglitz.

En este escenario de testimonios e informes sería de esperar un tono más conciliador de parte de La Casa Blanca, pero esto parece ser una esperanza perdida. Durante la víspera del foro de Davos, Bush, dirigiéndose al Congreso de mayoría republicana, remachó su teoría: "Si no hubiéramos actuado, el programa de desarrollo de armas de destrucción masiva del dictador continuaría hoy día. Norteamérica nunca pedirá autorización para defender la seguridad de sus ciudadanos. Nueve meses de largas negociaciones entre Estados Unidos y Gran Bretaña han logrado lo que no se hizo en doce años de trabajo diplomático con Irak. La tarea de construir un nuevo Irak es difícil y justa. Para todos los que aman la paz y la libertad un mundo sin el régimen de Saddam Hussein es un lugar mejor y más seguro. Los terroristas siguen conspirando contra Norteamérica y el mundo civilizado. Nuestra mayor responsabilidad es la defensa de los norteamericanos. Han pasado veintiocho meses desde el 11 septiembre 2001, más de dos años sin un solo ataque sobre nuestro suelo, pero es desacertado creer que el peligro quedó atrás. Este pensamiento es comprensible, tranquilizador, pero falso". (Le Monde, 21.01.04)

No hay signos de dolor o de arrepentimiento. Tampoco los hubo en el discurso del vicepresidente Cheney en Davos, quien vino a pedir 
a los europeos que se unan a su país en el esfuerzo común para combatir al terrorismo y a los regímenes que lo apoyan. "Europa no puede vivir como un enclave de paz rodeado de un mar de fanatismo y odio. Los europeos no deben verse obligados a elegir entre sus vocaciones europea y atlántica. Tenemos que hacer frente juntos a estos peligros. Nuestra cooperación entre nuestros gobiernos e instituciones internacionales son hoy día más importantes que en el pasado. El uso del poderío militar será siempre la decisión más difícil para un dirigente político, pero la debilidad y la vacilación pueden traer como consecuencia la tragedia. La seguridad no es divisible y la opción no es entre un mundo unipolar o multipolar, sino que la meta común es un mundo justo y democrático". Los corresponsales dicen que fue un discurso poco aplaudido, lleno de cinismo y no encajaba con el tono dominante en el programa de Davos. En ningún momento admitió crítica alguna a la guerra, cuyo inicio y desarrollo ha abierto una agria brecha entre Washington y sus socios europeos, Francia, Alemania y Rusia. Tampoco respondió al papel que jugaría la ONU en Irak ("me puedo meter en líos") y escurrió el bulto sobre "los presos de Guantánamo". Si en Davos 2004 no se han repetido las mutuas recriminaciones de 2003, "se ha pasado a un cierto escepticismo, pero la confianza no se ha recuperado". (El País, 23.01.04)

\section{Diálogo de civilizaciones}

Si los discursos de Bush al Congreso y de Richard Cheney en Davos alimentan la confrontación más que el acercamiento, los del presidente iraní, Mahomed Jatami, y del general Pervez Musharraf de Pakistán, vuelven a proponer el diálogo de civilizaciones, lanzando un puente entre Occidente y el mundo islámico. Jatami aseguró, a quien quiera oírle, que su gobierno nunca ha querido desarrollar o adquirir armas de destrucción masiva, algo a lo que de hecho "se opone de forma vehemente". Negó haber proporcionado armas nucleares a Corea del Norte y afirmó estar cooperando con la Agencia Internacional de la Energía Nuclear "en forma sincera y honesta". En su discurso abogó por el diálogo y el respeto mutuo para borrar toda sospecha de estar ligado con las cuatro palabras malditas: "armas de destrucción masiva". 
l’or su parte, Musharraf invitó al diálogo al presentar su "estrategia de la moderación ilustrada", que busca un equilibrio entre la amistad con Occidente y la fidelidad a los principios islámicos. "Todas las disputas políticas que el mundo está viviendo en los últimos años involucran a musulmanes y todos los musulmanes pueden ver en sus televisores el tratamiento que reciben sus hermanos de religión. Ahí está la raíz del profundo sentimiento de injusticia y privación", que perciben la mayoría de 1.300 millones de musulmanes. "Si a ello añadimos la pobreza y las bajas tasas de alfabetización que tenemos en el mundo islámico, tenemos un terreno abonado para los extremistas". Se trata de una estrategia bidireccional; el mundo islámico tiene que rechazar el extremismo y empujar el desarrollo económico y social. Occidente, por su parte, tiene que resolver todas las disputas políticas pendientes y asistir a los países islámicos en ese desarrollo socioeconómico". Musharraf repitió en varias ocasiones que el mundo islámico ya había empezado a dar pasos en la buena dirección y que ahora es "Occidente, Estados Unidos, a ONU, quienes tienen que ponerse manos a la obra". Musharraf reclamó de los países occidentales que "se corrija la asimetría económica y que los frutos de la globalización se distribuyan a favor de lo que no tienen nada", señalando que ahí está la raíz del extremismo que tanto alarma a Occidente. "La religión, el Islam, no tiene que ver nada con ese fenómeno. Existen dos errores de percepción: de un lado, en el mundo musulmán, se cree que el Islam está en el punto de mira; del otro, en Occidente se considera que el Islam es una religión militante, extremista e intolerante, contraria a la modernidad y al laicismo" (El País, 23.01.04)

Lo importante del discurso de Musharraf es que generó una seria reflexión de parte de hombres políticos y de negocios del mundo árabe, presentes en Davos. La falta de desarrollo económico en la región causa la decepción de la gente, su deriva al fundamentalismo musulmán y su inclinación al terrorismo. Todos están de acuerdo en el fracaso económico del mundo árabe. A pesar del petróleo los países árabes, en general, tienen un débil crecimiento económico, a veces inferior al crecimiento demográfico. A modo de ejemplo el triste caso de Arabia Saudita. "La pobreza genera desempleo en masa que afecta sobre todo a los jóvenes. ¿Qué hacer?" Debemos criticarnos a nosotros mismos en vez de culpar a los de fuera. No sabemos administrar 
nuestros negocios y nuestra falta de desarrollo proviene simplemente de nuestra débil competitividad. Todavía mantenemos elevadas barreras a la circulación de mercancías, hombres e ideas. ¿Qué clase de libertad económica hemos instalado? ¿Qué reforma en educación? ¿Qué ha hecho la sociedad civil a favor de la mujer? Los políticos tienen buenas intenciones, pero no saben qué hacer... Otros afirman que "las clases políticas árabes siguen siendo tradicionales". ¿Habrá un renacimiento? Se han propuesto una serie de medidas, insistiendo más en la educación, donde quedan lejos de otros países asiáticos y excomunistas. Por lo menos se ha lanzado un puente entre 'seguridad, prosperidad y cooperación'. El interrogante es ahora la esperada cooperación de Occidente. (Le Monde, 23.01.04)

Esto nos lleva a un tema concreto que ha quedado en la sala de espera: "remendar Doha". Hay que acercar las posturas comerciales tras los fracasos de Doha y Cancún, más los reclamos hechos en los foros de Bombay y Davos a los efectos negativos del comercio internacional. "Robert Zoellick había animado por carta a los ministros del ramo de los 146 países miembros de la OMC a retomar las negociaciones rotas en Doha, prometiendo hacer un serio esfuerzo para lograr un acuerdo que permita reducir o eliminar las tarifas aduaneras a la importación de bienes y servicios". (El País, 21.01.04) Por su parte, Lula de Silva, Jacques Chirac, Kofi Annan y Ricardo Lagos de Chile, han propuesto en Ginebra la creación de un fondo especial de lucha contra el hambre", financiado con una tasas sobre el comercio y ciertas ventas de armas y de determinadas transacciones financieras. (Le Monde, 31.01.04)

Haciendo un puente con el foro de Bombay, también en Davos tomaron la palabra cuatro reconocidos críticos de la globalización: Mary Robinson, alta comisionada de los derechos humanos de la ONU. Joseph Stiglitz, Deepark Nayyar, rector de la universidad de Nueva Dheli, y Juan Somavia, de la OIT. Para estos autores "el eje del mal es la pobreza, el sida y la guerra". El eje del mal gira al revés y arremolina a La Casa Blanca. Somavia dice: "Existen dos visiones de la prosperidad: allá abajo, la prosperidad es la próxima comida, aquí el crecimiento económico; allá abajo la seguridad es tener un empleo, aquí es el terrorismo". Mary Robinson no se hace muchas ilusiones sobre los cambios en Davos: "Lo que me preocupa es la acumulación de tanto poder en tan pocas manos". (Le Monde, 26.01.04) 
Los corresponsales del diario El País cierran su comentario diciendo que "los empresarios dejan Davos con dudas sobre la recuperación económica, con mucha cautela sobre la recuperación económica mundial y sin convencimiento de que los desequilibrios de Estados Unidos (déficits gemelos del presupuesto y de la cuenta corriente de la balanza de pagos) puedan sostenerse mucho tiempo". Importante es la otra sensación: "Los líderes europeos, además, comienzan a ver con recelo a Davos como un foro de EEUU. Los organizadores del Foro Económico Mundial son conscientes de que la presencia de hombres importantes de la Administración de Bush en los últimos dos encuentros puede enajenar definitivamente la disposición de presidentes y primeros ministros europeos a comprometer su asistencia". (El País, 26.01.04) No sólo ha sido en los dos últimos foros de Davos, sino también en la última cumbre de Naciones Unidas, donde el entrampamiento de la coalición en la guerra de Irak sacó de la agenda los puntos claves de la reforma de Naciones Unidas y las respuestas viables a las 'amenazas duras y blandas' propuestas por K. Annan. Por su parte, Europeos y Norteamericanos deben pensar que su comportamiento imperial en las cumbres de Doha y Cancún pueden empujar al mundo hacia una "globalización regresiva". La sombra de Bombay, ise ha proyectado sobre el foro de Davos? 